\title{
Detection of Muscle Activities in the sEMG Signal by Using Frequency Features and Adaptive Decision Threshold
}

\author{
Husamuldeen Khalid Hameed ${ }^{1 *}$, Wan Zuha Wan Hasan ${ }^{1,2}$, Suhaidi Shafie ${ }^{2}$, Siti \\ Anom Ahmad ${ }^{1}$ and Haslina Jaafar ${ }^{1}$ and Liyana Najwa Inche Mat ${ }^{3}$
}

${ }^{1}$ Department of Electrical and Electronic Engineering, Faculty of Engineering, Universiti Putra Malaysia, 43400 UPM Serdang, Selangor, Malaysia

${ }^{2}$ Institute of Advanced Technology (ITMA), Universiti Putra Malaysia, 43400 UPM Serdang, Selangor, Malaysia ${ }^{3}$ Department of Medicine, Faculty of Medicine and Health Sciences, Universiti Putra Malaysia, 43400 UPM Serdang, Selangor, Malaysia

\begin{abstract}
Reliable detection of muscle activities from the surface electromyography (sEMG) signal is an important factor that makes the sEMG controlled orthotic devices a practical tool for assisting disabled people. In spite of the advantages of employing the sEMG signal as a control signal, the changes in the amplitude characteristics of this signal due to many factors and consequent variations in the required decision threshold may impede this control paradigm from being a reliable control method for such devices. Therefore, the performance of the algorithms intended to detect muscle activities should be immune against the involuntary amplitude variations of the sEMG signal. Moreover, the decision threshold value must be adaptive to the changes in the sEMG signal characteristics to reduce the number of false alarms that may arise with the fixed threshold and lead to

\section{ARTICLE INFO}

\section{Article history:}

Received: 10 February 2020

Accepted: 13 November 2020

Published: 31 December 2020

DOI: https://doi.org/10.47836/pjst.28.S2.01

E-mail addresses:

husamuldeen72@gmail.com (Husamuldeen Khalid Hameed)

wanzuha@upm.edu.my (Wan Zuha Wan Hasan)

suhaidi@upm.edu.my (Suhaidi Shafie)

sanom@upm.edu.my (Siti Anom Ahmad)

jhaslina@upm.edu.my (Haslina Jaafar)

liyananajwa@upm.edu.my (Liyana Najwa Inche Mat)

*Corresponding author

unintended movements to these devices. In this paper, an amplitude-independent algorithm had been developed with an adaptive decision threshold; the algorithm employed only frequency features of the sEMG signal to detect muscle activities. These features are the previously developed Adaptive Zero Crossing feature and the new proposed Adaptive Wilson Amplitude feature. The Mean Instantaneous Frequency value of the sEMG signal was used as an
\end{abstract}


adaptive decision threshold value to improve the detection performance and to minimize the number of false alarms produced with the utilization of inappropriate fixed decision threshold value. A comparison with an amplitude-independent algorithm that employed fixed decision threshold had revealed an improved performance regarding the resistance against false alarms.

Keywords: Adaptive decision threshold, false alarms, frequency features, muscle activity detection, sEMG

\section{INTRODUCTION}

Control of orthotic and prosthetic robotic devices by employing sEMG signals has acquired a lot of attention due to the advantages of this control method. One of the most important advantages is the naturalness, because this control method requires the same type of muscle activities used to move the limb which makes the control more natural (Meeker et al., 2017). Other advantages like simplicity in obtaining sEMG signal by surface electrodes as well as the little time delay between human intent and the real movement of the device (Rosen et al., 2001) have made this control method the most common type used to operate orthotic devices (Lobo-Prat et al., 2014). However, robust sEMG control is still a challenging process because of the variations in the amplitude characteristics of the sEMG signal due to many factors. Factors like electrode location, electrode movement, skin electrical impedance, fatigue, and sweat are affecting the amplitude of the sEMG over the time (Huang et al., 2015; Marchal-Crespo et al., 2009). Moreover, the amplitudes of the sEMG signals are not constant during the recording time due to the variations in the characteristics of the electrode-skin interface and due to the changes in the level of the ground reference signal (Severini et al., 2012). Therefore, the algorithms intended to detect muscle activities should be resistant against the involuntary amplitude variations of the signal and should have an adaptive decision threshold that adapts the algorithm detection to the variations in the sEMG signal level.

In the literature, various algorithms have been proposed to detect muscle activities from the sEMG signal. The conventional algorithms compare the amplitude features of the signal with a predefined threshold value, this threshold must be determined according to the amplitude of the measured signal during no activity period and must be modified whenever this amplitude changes. More complicated algorithms have been developed to overcome the problem of the predefined fixed threshold. Xu et al. (2013) had developed a detection algorithm based on the maximum likelihood method improved by an adaptive threshold, but this method depended on the signal amplitude features to report muscle activities. Yang et al. (2017) had enhanced the detection algorithm based on the Teager Kaiser Energy (TKE) operator by employing two image technologies to detect the amplitude variations in the 
weak and noisy sEMG signal. Zhang and Zhan (2012) had developed a muscle activity detection algorithm against spurious background spikes by using sample entropy analysis of the sEMG signal, but this method required high computation efforts and therefore it was difficult to be implemented in real time. Moreover, it needed to preset threshold value for the muscle activity onset detection. Liu and Liu (2016) had developed a detection algorithm by employing the integrated profile of the sEMG signal in the presence of the spurious background spikes for the spinal cord injury patients, but this method depended on the changes in the amplitude of the sEMG signal to report the muscle activities. By using the continuous wavelet transform, Merlo et al. (2003) had developed a detection algorithm to detect the amplitude variations of the sEMG signal in order to report the muscle activities. Semmaoui et al. (2012) had developed an adaptive threshold for the Smoothed Teager Energy Operator detection method, but this method was amplitude dependent. Severini et al. (2012) had improved the Bonato double threshold detection method by making the decision threshold value adaptive to the variations in the sEMG signal to noise ratio, but still this method depended on the amplitude characteristics to report the muscle activities.

To our knowledge, only three amplitude-independent studies (D'Anna et al., 2019; Hameed et al., 2017, 2018) have been proposed in the literature to detect sEMG muscle activities, where the study in D'Anna et al. (2019) was an improvement to the algorithm presented in Hameed et al. (2017), but all these studies are with fixed decision threshold. In this paper, an amplitude-independent algorithm with an adaptive decision threshold value is proposed to detect muscle activities. The algorithm has the ability to detect muscle activities that have a low signal to noise ratio (SNR). The algorithm employs two frequency features in the detection process which are the Adaptive Zero Crossing (Hameed et al., 2017) and the new proposed feature which is the Adaptive Wilson Amplitude. In contrast to its name, the Wilson Amplitude is a frequency feature of the sEMG signal (Phinyomark et al., 2012). The algorithm utilizes the Mean Instantaneous Frequency of the sEMG signal as an adaptive decision threshold value to report muscle activities. It is hypothesized that the employment of the proposed Wilson feature will enhance the muscle activity detection process, as well as the use of the adaptive threshold, will reduce the number of false alarms compared to the fixed threshold algorithm presented in (Hameed et al., 2017). This research tests this hypothesis.

\section{MATERIALS AND METHOD}

The sEMG signal from the forearm muscle responsible for fingers flexion (Flexor Digitorum Superficialis muscle) of a healthy subject was obtained and amplified by using a muscle sensor (MyoWare from the Advancer Technology). The subject was ordered to generate a weak muscle activity every about two seconds, then to generate one weak muscle activity about every five seconds, then to stop generating muscle activities. The analog sEMG 
signal was sampled at 1000 samples per second and converted to a digital signal by using an analog to digital converter (NI USB-6001 DAQ from National Instruments). The digital input samples were grouped into segments of 100 samples per segment and the proposed muscle activity detection algorithm developed by using LabVIEW software was applied in real time for each segment. The hardware setup used to conduct the experiments is illustrated in Figure 1. Figure 2 shows the flow diagram of the proposed algorithm.

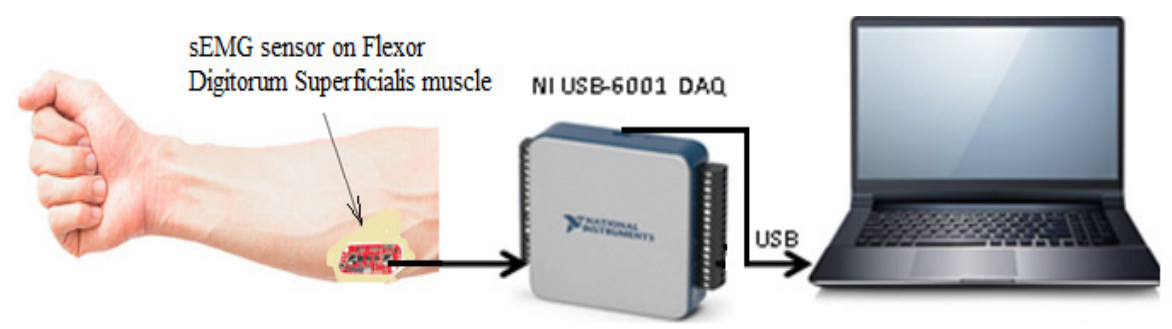

Figure 1. The hardware setup

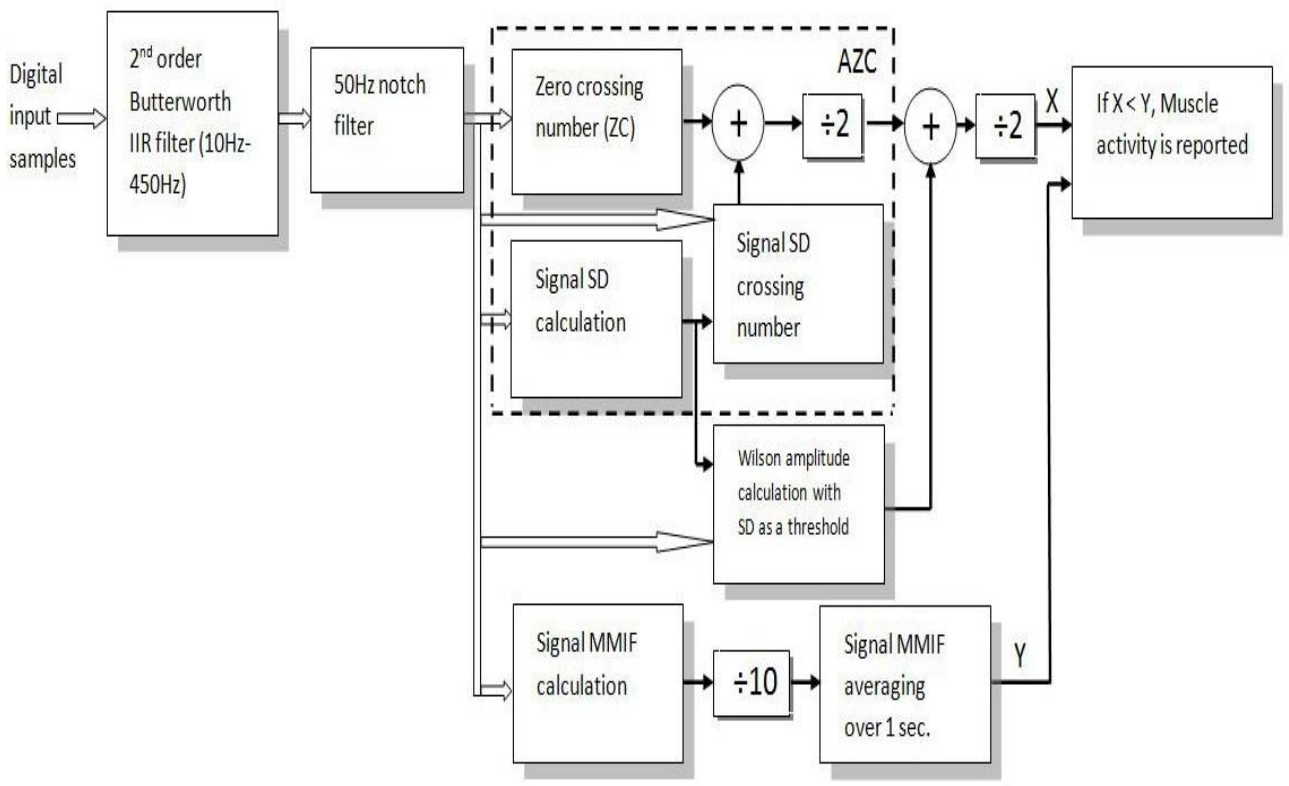

Figure 2. Muscle activity detection algorithm

A Bandpass filter was used to attenuate the artifact and high frequency noises, and then a notch filter was employed to remove the power line interference. The proposed Adaptive Wilson Amplitude (AWA) feature was extracted for each segment and averaged with the 
Adaptive Zero Crossing (AZC) feature (dotted line in Figure 2). The result was compared with the adaptive threshold value; a muscle activity was declared if the (AZC+AWA)/2 value was less than the threshold value. To compute the adaptive decision threshold value, the Mean Instantaneous Frequency values for each segment were calculated by using the Gabor Transform, and then the mean of the Mean Instantaneous Frequency values (MMIF) for each segment was computed. The MMIF value is divided by 10 in order to make it within the same range of AZC and AWA values. These (MMIF/10) values were averaged over one second during no muscle activity period to represent the adaptive decision threshold value. The one second period is selected as a compromise between greater periods which leads to reduce adaptation ability and smaller periods which leads to reduce flatness of the threshold line, where this reduction in the flatness increases the possibility of misdetection and false alarms. Since the MMIF values were decreased during muscle activity periods, the averaging process took the last average (MMIF/10) value of the no muscle activity period as a threshold value during muscle activity period to prevent dropping in the threshold line. The proposed algorithm is adaptive and does not need to preset any value before the operation.

The proposed $A W A$ feature was computed by using the signal Standard Deviation (SD) for each segment as a new threshold value instead of the fixed threshold value used in the traditional Wilson Amplitude feature (WA). This new threshold makes the $A W A$ value independent of the sEMG signal amplitude and does not need to preset a threshold value based on the signal amplitude during no activity period. The proposed $A W A$ feature is defined for each segment as:

$$
\begin{aligned}
& A W A=\sum_{n=0}^{N-1} \quad f(|x(n)-x(n-1)|) \\
& \text { where } f(x)= \begin{cases}1 & \text { if } x \geq S D \\
0 & \text { otherwise }\end{cases}
\end{aligned}
$$

where $x(n)$ is input samples, $N$ is the number of samples in each segment, $S D$ is the standard deviation of the segment and it is calculated as:

$$
S D=\sqrt{\left(\sum_{n=0}^{N-1}(x(n)-\mu)^{2}\right) / N}
$$

where $\mu$ is the segment mean value and it is calculated as:

$$
\mu=\left(\sum_{n=0}^{N-1} x(n)\right) / N
$$

The $A Z C$ value for each segment is calculated by averaging the two values, $Z C$ and $S D C$, as $(Z C+S D C) / 2$ (Hameed et al., 2017), where $Z C$ value of the segment (number of times that sEMG signal crosses the zero volt line) is calculated as: 


$$
Z C=\sum_{n=0}^{N-1} \quad f(x(n) \times x(n-1))
$$

where $f(x)=\left\{\begin{array}{lc}1 & \text { if } x \leq 0 \\ 0 & \text { otherwise }\end{array}\right.$

the $S D C$ value of the segment (number of times that sEMG signal crosses the standard deviation of the segment) is calculated as:

$$
\begin{aligned}
& S D C=\sum_{n=0}^{N-1} \quad f([x(n)-S D] \times[x(n-1)-S D]) \\
& \text { where } f(x)= \begin{cases}1 & \text { if } x \leq 0 \\
0 & \text { otherwise }\end{cases}
\end{aligned}
$$

\section{RESULTS}

Figure 3 clarifies the difference in the behavior of AWA and WA values when the muscle activities are present. The AWA value decreased when the muscle activity was present,

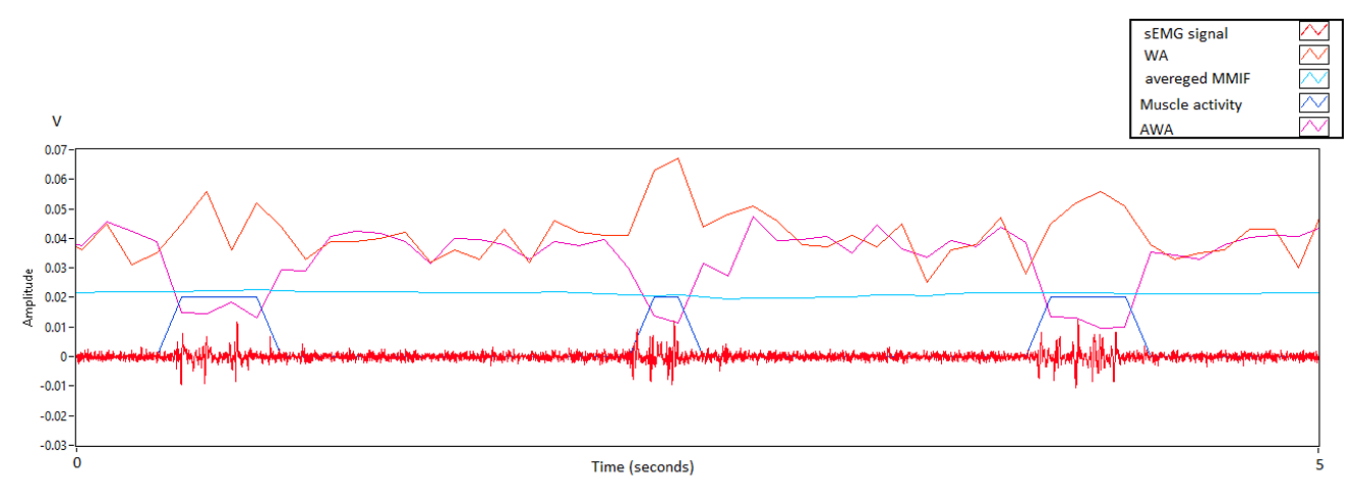

while WA value increased with the presence of muscle activity.

Figure 3. Comparison of the WA value (fixed threshold) and AWA value (SD as a threshold) for sEMG signal with three muscle activities (presented by the blue lines); WA, AWA and averaged MMIF are divided by 1000 to enable presenting in the same chart with the sEMG signal.

Averaging of AWA value with the AZC value was used to enhance the muscle activity detection process as shown in Figure 4, were employing the AZC alone had failed to detect the muscle activity. Moreover, the averaging process enhanced muscle activity detection in noisy sEMG signals as illustrated in Figure 5. Furthermore, the averaging enhanced muscle activity detection time and made it more accurate because the average value was more precise for detecting the period of muscle activity as clarified in Figure 6. 


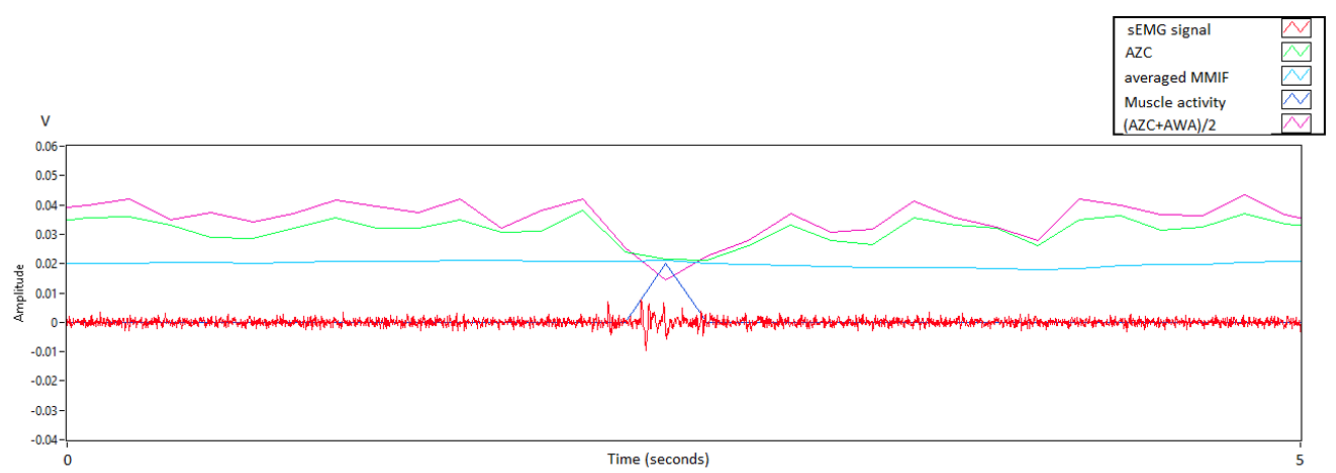

Figure 4. sEMG signal with one muscle activity (presented by the blue line); Employing of the (AZC+AWA)/2 instead of AZC alone enhances the muscle activity detection. AZC, (AZC+AWA)/ 2 and averaged MMIF are divided by 1000 to enable presenting in the same chart with the sEMG signal

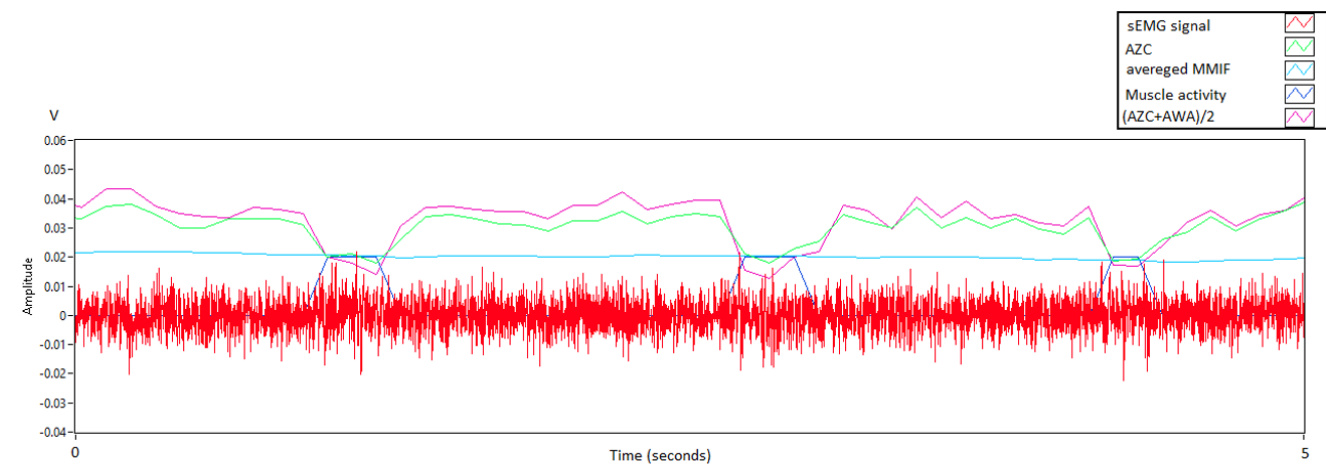

Figure 5. Noisy sEMG signal with three muscle activities (presented by the blue lines); Employing of the $(\mathrm{AZC}+\mathrm{AWA}) / 2$ enhances the detection of muscle activities in the noisy signal. AZC, (AZC+AWA)/2 and averaged MMIF are divided by 1000 to enable presenting in the same chart with the sEMG signal

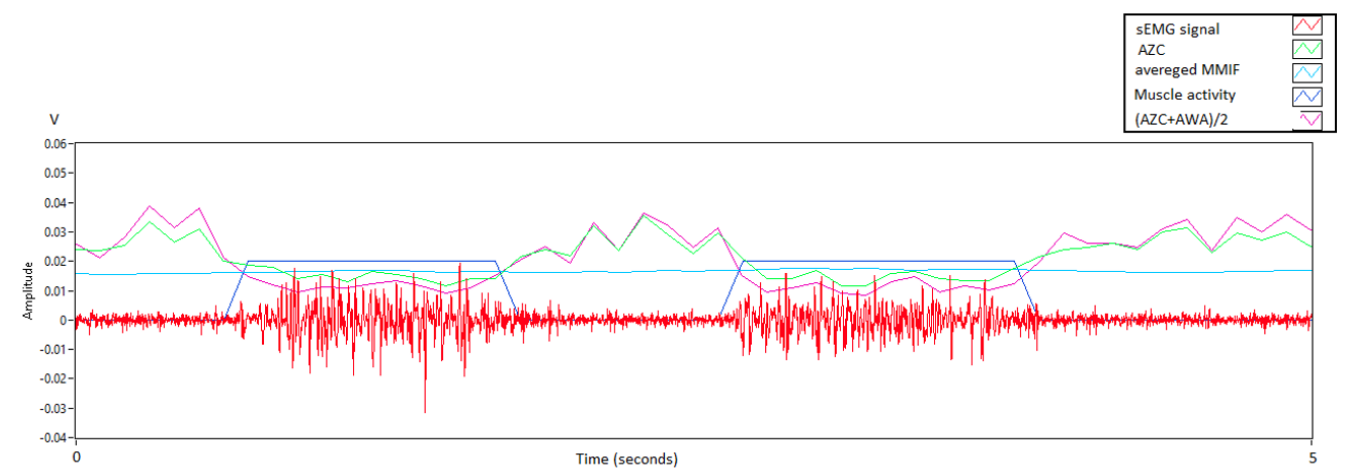

Figure 6. sEMG signal with two muscle activities (presented by the blue lines); Employing of the (AZC+AWA)/2 improves the accuracy of the muscle activity detection. AZC, (AZC+AWA) $/ 2$ and averaged MMIF are divided by 1000 to enable presenting in the same chart with the sEMG signal 
Utilization of the averaged MMIF value as an adaptive decision threshold value reduces the possibility of generating false alarms. The adaptive threshold value decreased when the sEMG signal was contaminated by low frequency noise components as illustrated in Figure 7 and increased when the signal was contaminated by high frequency noise components as shown in Figure 8. This adaptation makes the threshold value to stay as close as to the $(\mathrm{AZC}+\mathrm{AWA}) / 2$ value in order to detect muscle activities and far enough to avoid false alarms that may arise due to the presented noise components.

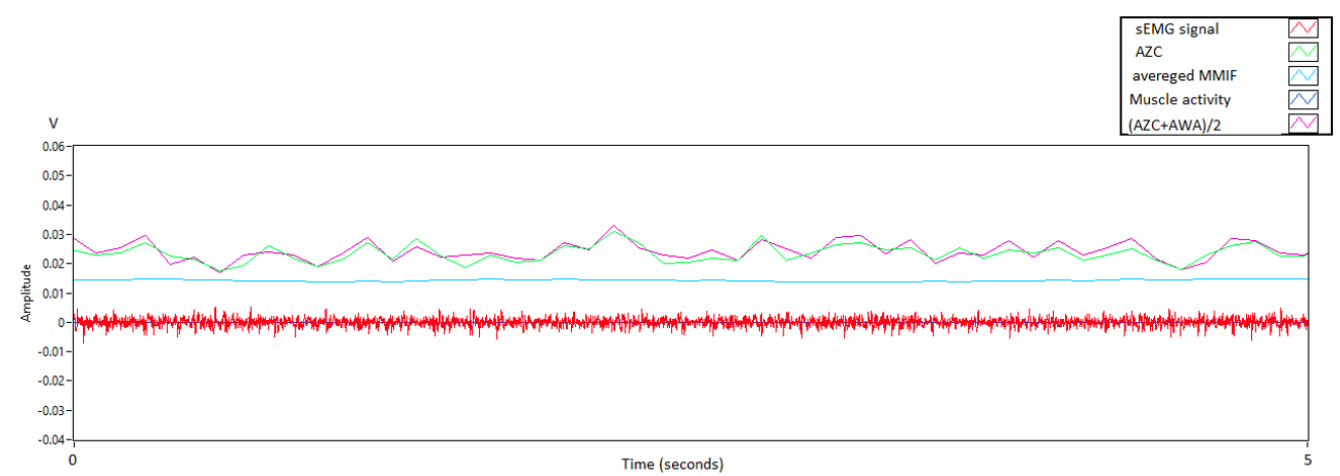

Figure 7. sEMG signal without muscle activities, the adaptive threshold value is about 15 ; AZC, (AZC+AWA)/2 and averaged MMIF are divided by 1000 to enable presenting in the same chart with the sEMG signal

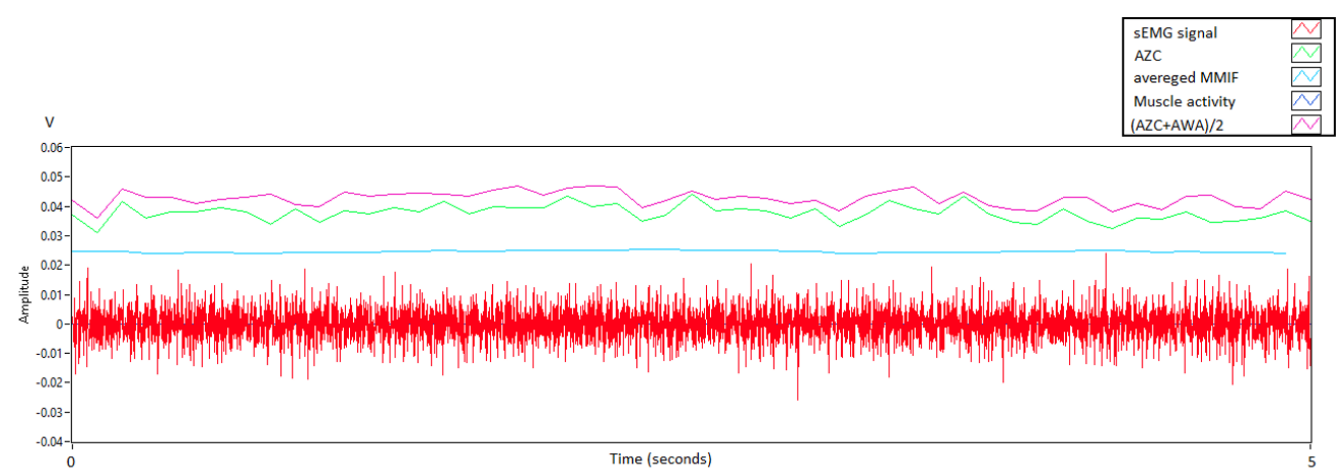

Figure 8. sEMG signal without muscle activities, the adaptive threshold value is about 25; AZC, (AZC+AWA)/2 and averaged MMIF are divided by 1000 to enable presenting in the same chart with the sEMG signal

\section{DISCUSSION}

Employing the proposed AWA feature as well as the adaptive decision threshold value had enhanced the muscle activity detection process because the average value (AZC+AWA)/2 was less than the AZC value during muscle activity period which increased the possibility 
of detection as shown in Figures 4, 5 and 6. Moreover, the averaging process minimized the possibility of false alarms because the average value was greater than AZC value during no muscle activity period and therefore it was more distant from the threshold value.

The previous algorithm presented in Hameed et al. (2017) had employed the MMIF of the signal to enhance the detection process by multiplying the AZC value with the normalized MMIF value as $((M M I F / 200) \times A Z C)$; the previous algorithm assumed that the average value of MMIF was $200 \mathrm{~Hz}$ during no activity period, therefore it divided the MMIF by 200 to normalize it. Due to the imposed low or high frequency noise components, this process may lead to false alarms when the average value of MMIF is not $200 \mathrm{~Hz}$. Therefore, the proposed algorithm employs the MMIF feature as an adaptive decision threshold value by averaging the MMIF values over one second during no activity periods. This averaged value is used as a decision threshold value adapted to the variations in the sEMG signal characteristics as shown in Figures 7 and 8. Moreover, the previous algorithm employs a fixed decision threshold value to decide whether a muscle activity is reported or not, this process leads to false alarms when an inappropriate threshold value is chosen, consequently, the fixed threshold value must be predefined properly according to sEMG signal characteristics. In contrast, the proposed algorithm does not need to predefine a threshold value; it is defined automatically according to the MMIF of the sEMG signal.

A comparison was conducted by using real sEMG signal between the fixed threshold algorithm presented in Hameed et al. (2017) and the proposed adaptive threshold algorithm with respect to the resistance against false alarms (threshold value of 25 was chosen for the previous algorithm as was selected in Hameed et al. (2017). Since the sEMG signal used in the experiment was contaminated by low frequency noise components, the previous algorithm had produced many false alarms because of the high fixed threshold value of 25 . In contrast, the proposed algorithm did not generate any false alarm due to the advantage of employing adaptive decision threshold as illustrated in Figure 9.

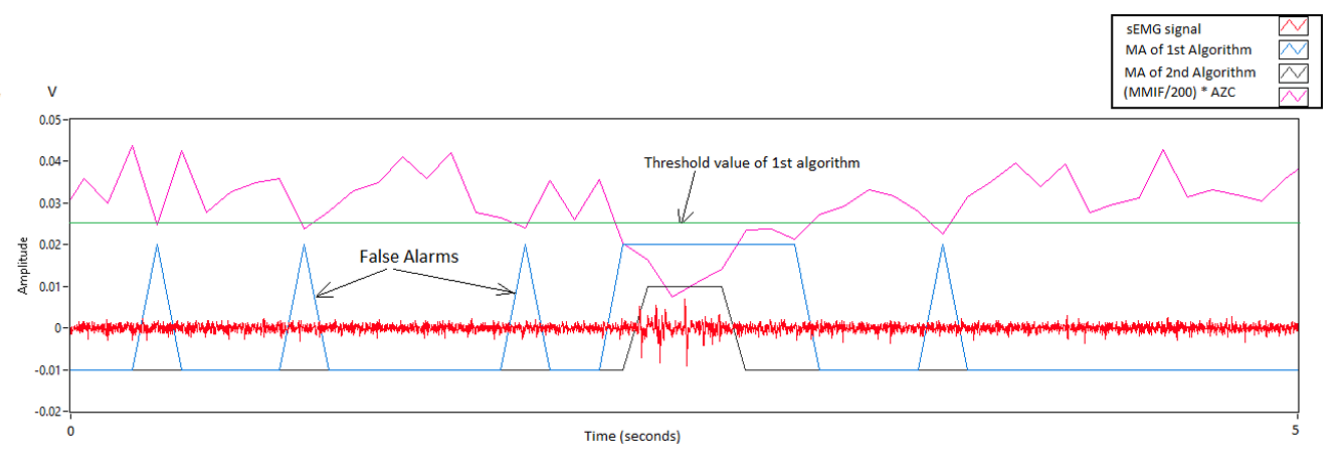

Figure 9. sEMG signal with one muscle activity (presented by the black line); A comparison between the performance of the previous algorithm (1st algorithm presented in [13] Hameed et al., 2017) and the proposed algorithm (2nd) regarding the resistance against false alarms. (MMIF/200) $\times \mathrm{AZC}$ and the threshold value are divided by 1000 to enable presenting in the same chart with the sEMG signal. MA: Muscle Activity 


\section{CONCLUSIONS}

An improved muscle activity detection algorithm with a new proposed frequency feature and adaptive decision threshold value has been successfully developed. The algorithm is amplitude-independent because it employs only frequency features to report muscle activities from the sEMG signal. In addition to the previously developed Adaptive Zero Crossing (AZC) feature, the algorithm employs the newly developed Adaptive Wilson Amplitude feature (AWA) to enhance the muscle activity detection process and improve resistance against false alarms. The algorithm employs an adaptive threshold value based on the Mean Instantaneous Frequency of the sEMG signal to improve the detection process and to reduce false alarms that may arise when unsuitable fixed threshold value is used. The efficacy of the algorithm has been evaluated in real time on a healthy subject by using the sEMG signal collected from the forearm muscle responsible for finger flexion (Flexor Digitorum Superficialis). A comparison between the proposed algorithm and the amplitudeindependent algorithm that has a fixed decision threshold presented in Hameed et al., (2017) showed improved performance for the proposed algorithm in terms of resistance against false alarms.

For future work, the efficacy of the proposed algorithm will be verified on stroke patients to enable the utilization of this algorithm for controlling robotic devices devoted to disabled people.

\section{ACKNOWLEDGEMENT}

The authors would like to thank the Ministry of Education, Malaysia for supporting and funding this study under the MOE-FRGS scheme with grant number of 03-01-17-1893FR.

\section{REFERENCES}

D’Anna, C., Varrecchia, T., Schmid, M., \& Conforto, S. (2019). Using the frequency signature to detect muscular activity in weak and noisy myoelectric signals. Biomedical Signal Processing and Control, 52, 69-76. doi:10.1016/j.bspc.2019.02.026

Hameed, H., Hassan, W., Shafie, S., Ahmad, S., \& Jaafar, H. (2017, August, 23-25). An amplitude independent muscle activity detection algorithm based on adaptive zero crossing technique and mean instantaneous frequency of the sEMG signal. In IEEE Regional Symposium on Micro and Nanoelectronics (RSM). Batu Ferringhi, Malaysia.

Hameed, H., Hassan, W., Shafie, S., Ahmad, S., \& Jaafar, H. (2018, November 28-30). Soft robotic glove system controlled with amplitude independent muscle activity detection algorithm by using single sEMG channel. In IEEE 5th International Conference on Smart Instrumentation, Measurement and Applications (ICSIMA 2018). Songkhla, Thailand. 
Huang, J., Huo, W., Xu, W., Mohammed, S., \& Amirat, Y. (2015). Control of upper-limb power-assist exoskeleton using a human-robot interface based on motion intention recognition. IEEE Transactions on Automation Science and Engineering, 12(4), 1257-1270. doi: 10.1109/tase.2015.2466634

Liu, J., \& Liu, Q. (2016). Use of the integrated profile for voluntary muscle activity detection using EMG signals with spurious background spikes: A study with incomplete spinal cord injury. Biomedical Signal Processing and Control, 24, 19-24. doi:10.1016/j.bspc.2015.09.004

Lobo-Prat, J., Kooren, P., Stienen, A., Herder, J., Koopman, B., \& Veltink, P. (2014). Non-invasive control interfaces for intention detection in active movement-assistive devices. Journal of NeuroEngineering and Rehabilitation, 11(1), 1-22. doi: 10.1186/1743-0003-11-168

Marchal-Crespo, L., \& Reinkensmeyer, D. (2009). Review of control strategies for robotic movement training after neurologic injury. Journal of NeuroEngineering and Rehabilitation, 6(1), 1-15. doi: 10.1186/17430003-6-20

Meeker, C., Park, S., Bishop, L., Stein, J., \& Ciocarlie, M. (2017, July, 17-20). EMG pattern classification to control a hand orthosis for functional grasp assistance after stroke. In International Conference on Rehabilitation Robotics (ICORR) (pp. 1203-1210). QEII Centre, London, UK.

Merlo, A., Farina, D., \& Merletti, R. (2003). A fast and reliable technique for muscle activity detection from surface EMG signals. IEEE Transactions on Biomedical Engineering, 50(3), 316-323. doi: 10.1109/ tbme.2003.808829

Phinyomark, A., Phukpattaranont, P., \& Limsakul, C. (2012). Feature reduction and selection for EMG signal classification. Expert Systems with Applications, 39(8), 7420-7431. doi: 10.1016/j.eswa.2012.01.102

Rosen, J., Brand, M., Fuchs, M. B. \& Arcan, M. (2001). A myosignal-based powered exoskeleton system. IEEE Transactions on Systems, Man and Cybernetics, Part A: Systems and Humans, 31(3), 210-222. doi: $10.1109 / 3468.925661$

Semmaoui, H., Drolet, J., Lakhssassi, A., \& Sawan, M. (2012). Setting adaptive spike detection threshold for smoothed TEO based on robust statistics theory. IEEE Transactions on Biomedical Engineering, 59(2), 474-482. doi: 10.1109/tbme.2011.2174992

Severini, G., Conforto, S., Schmid, M., \& D’Alessio, T. (2012). Novel formelation of a double threshold algorithm for estimation of muscle activation intervals designed for variable SNR environments. Journal of Electromyography and Kinesiology, 22(6), 878-885. doi: 10.1016/j.jelekin.2012.04.010

Xu, Q., Quan, Y., Yang, L., \& He, J. (2013). An adaptive algorithm for the determination of the onset and offset of muscle contraction by EMG signal processing. IEEE Transactions on Neural Systems and Rehabilitation Engineering, 21(1), 65-73. doi:10.1109/tnsre.2012.2226916

Yang, D., Zhang, H., Gu, Y., \& Liu, H. (2017). Accurate EMG onset detection in pathological, weak and noisy myoelectric signals. Biomedical Signal Processing and Control, 33, 306-315. doi: 10.1016/j. bspc.2016.12.014

Zhang, X., \& Zhan, P. (2012). Sample entropy analysis of surface EMG for improved muscle activity onset detection against spurious background spikes. Journal of Electromyography and Kinesiology, 22(6), 901-907. doi: 10.1016/j.jelekin.2012.06.005 
